\title{
Molecular Hydrogen as an Emerging Therapeutic Medical Gas for Neurodegenerative and Other Diseases
}

\author{
Kinji Ohno, ${ }^{1}$ Mikako Ito, ${ }^{1}$ Masatoshi Ichihara, ${ }^{2}$ and Masafumi Ito ${ }^{3}$ \\ ${ }^{1}$ Division of Neurogenetics, Center for Neurological Diseases and Cancer, Nagoya University Graduate School of Medicine, \\ 65 Tsurumai, Showa-ku, Nagoya 466-8550, Japan \\ ${ }^{2}$ Department of Biomedical Sciences, College of Life and Health Sciences, Chubu University, Aichi 487-8501, Japan \\ ${ }^{3}$ Research Team for Mechanism of Aging, Tokyo Metropolitan Institute of Gerontology, Tokyo 173-0015, Japan \\ Correspondence should be addressed to Kinji Ohno, ohnok@med.nagoya-u.ac.jp
}

Received 11 January 2012; Revised 24 March 2012; Accepted 13 April 2012

Academic Editor: Marcos Dias Pereira

Copyright ( 2012 Kinji Ohno et al. This is an open access article distributed under the Creative Commons Attribution License, which permits unrestricted use, distribution, and reproduction in any medium, provided the original work is properly cited.

Effects of molecular hydrogen on various diseases have been documented for 63 disease models and human diseases in the past four and a half years. Most studies have been performed on rodents including two models of Parkinson's disease and three models of Alzheimer's disease. Prominent effects are observed especially in oxidative stress-mediated diseases including neonatal cerebral hypoxia; Parkinson's disease; ischemia/reperfusion of spinal cord, heart, lung, liver, kidney, and intestine; transplantation of lung, heart, kidney, and intestine. Six human diseases have been studied to date: diabetes mellitus type 2, metabolic syndrome, hemodialysis, inflammatory and mitochondrial myopathies, brain stem infarction, and radiation-induced adverse effects. Two enigmas, however, remain to be solved. First, no dose-response effect is observed. Rodents and humans are able to take a small amount of hydrogen by drinking hydrogen-rich water, but marked effects are observed. Second, intestinal bacteria in humans and rodents produce a large amount of hydrogen, but an addition of a small amount of hydrogen exhibits marked effects. Further studies are required to elucidate molecular bases of prominent hydrogen effects and to determine the optimal frequency, amount, and method of hydrogen administration for each human disease.

\section{Introduction}

Molecular hydrogen $\left(\mathrm{H}_{2}\right)$ is the smallest gas molecule made of two protons and two electrons. Hydrogen is combustible when the concentration is $4-75 \%$. Hydrogen, however, is a stable gas that can react only with oxide radical ion $\left(\bullet \mathrm{O}^{-}\right)$ and hydroxyl radical $(\bullet \mathrm{OH})$ in water with low reaction rate constants [1]:

$$
\begin{array}{cc}
\bullet \mathrm{O}^{-}+\mathrm{H}_{2} \longrightarrow \mathrm{H} \bullet+\mathrm{OH}^{-} & k=8.0 \times 10^{7} \mathrm{M}^{-1} \cdot \mathrm{s}^{-1} \\
\bullet \mathrm{OH}+\mathrm{H}_{2} \longrightarrow \mathrm{H} \bullet+\mathrm{H}_{2} \mathrm{O} & k=4.2 \times 10^{7} \mathrm{M}^{-1} \cdot \mathrm{s}^{-1} \\
\mathrm{H} \bullet+\bullet \mathrm{OH} \longrightarrow \mathrm{H}_{2} \mathrm{O} & k=7.0 \times 10^{9} \mathrm{M}^{-1} \cdot \mathrm{s}^{-1} .
\end{array}
$$

The reaction rate constants of $\bullet \mathrm{O}^{-}$and $\bullet \mathrm{OH}$ with other molecules are mostly in the orders of $10^{9}$ to $10^{10} \mathrm{M}^{-1} \cdot \mathrm{s}^{-1}$, whereas those with $\mathrm{H}_{2}$ are in the order of $10^{7} \mathrm{M}^{-1} \cdot \mathrm{s}^{-1}$. Hydrogen, however, is a small molecule that can easily dissipate throughout the body and cells, and the collision rates of hydrogen with other molecules are expected to be very high, which is likely to be able to overcome the low reaction rate constants [2]. Hydrogen is not easily dissolved in water, and 100\%-saturated hydrogen water contains $1.6 \mathrm{ppm}$ or $0.8 \mathrm{mM}$ hydrogen at room temperature.

In 1995, hydrogen was first applied to human to overcome high-pressure nervous syndrome in deep sea diving [3]. Hydrogen was used to reduce nitrogen $\left(\mathrm{N}_{2}\right)$ toxicity and to reduce breathing resistance in the deep sea. In 2001, being prompted by the radical-scavenging activity of hydrogen, Gharib and colleagues examined an effect of molecular hydrogen on a mouse model of schistosomiasisassociated chronic liver inflammation [4]. Mice were placed in a chamber with $70 \%$ hydrogen gas for two weeks. The mice exhibited decreased fibrosis, improvement of hemodynamics, increased nitric oxide synthase (NOS) II activity, 


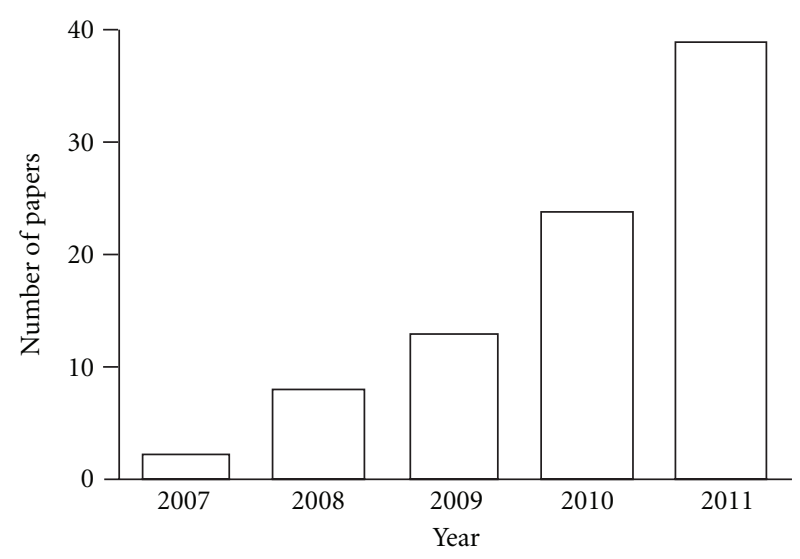

FIGURE 1: Number of papers that report effects of molecular hydrogen since 2007 shown in Table 1.

increased antioxidant enzyme activity, decreased lipid peroxide levels, and decreased circulating tumor-necrosis-factor(TNF-) $\alpha$ levels. Although helium gas also exerted some protective effects in their model, the effect of helium gas was not recapitulated in a mouse model of ischemia/reperfusion injury of the liver [5].

\section{Effects of Hydrogen Have Been Reported in 63 Disease Models and Human Diseases}

A major breakthrough in hydrogen research occurred after Ohsawa and colleagues reported a prominent effect of molecular hydrogen on a rat model of cerebral infarction in June 2007 [6]. Rats were subjected to left middle cerebral artery occlusion. Rats placed in $2-4 \%$ hydrogen gas chamber showed significantly smaller infarction volumes compared to controls. They attributed the hydrogen effect to the specific scavenging activity of hydroxyl radical $(\bullet \mathrm{OH})$. They also demonstrated that hydrogen scavenges peroxynitrite $\left(\mathrm{ONOO}^{-}\right)$but to a lesser extent.

As have been previously reviewed $[7,8]$, effects of molecular hydrogen on various diseases have been reported since then. The total number of disease models and human diseases for which molecular hydrogen has been proven to be effective has reached 63 (Table 1). The number of papers is increasing each year (Figure 1). Among the 87 papers cited in Table 1, 21 papers showed an effect with inhalation of hydrogen gas, 23 with drinking hydrogen-rich water, 27 with intraperitoneal administration or drip infusion of hydrogen-rich saline, 10 with hydrogen-rich medium for cell or tissue culture, and 6 with the other administration methods including instillation and dialysis solution. In addition, among the 87 papers, 67 papers showed an effect in rodents, 7 in humans, 1 in rabbits, 1 in pigs, and 11 in cultured cells or cultured tissues.

Two papers, however, showed that hydrogen was ineffective for two disease models (Table 2). One such disease was moderate to severe neonatal brain hypoxia [9], although marked effects of hydrogen gas $[10,11]$ and intraperitoneal administration of hydrogen-rich saline [12] on neonatal brain hypoxia have been reported in rats $[10,12]$ and pigs [11]. We frequently observe that therapeutic intervention that is effective for mild cases has little or no effect on severe cases, and hydrogen is unlikely to be an exception. Another disease is muscle disuse atrophy [13]. Although oxidative stress is involved in the development of muscle disuse atrophy, oxidative stress may not be a major driving factor causing atrophy and thus attenuation of oxidative stress by hydrogen may not be able to exhibit a beneficial effect.

Effects of molecular hydrogen have been observed essentially in all the tissues and disease states including the brain, spinal cord, eye, ear, lung, heart, liver, kidney, pancreas, intestine, blood vessel, muscle, cartilage, metabolism, perinatal disorders, and inflammation/allergy. Among them, marked effects are observed in ischemia/reperfusion disorders as well as in inflammatory disorders. It is interesting to note, however, that only three papers addressed effects on cancers. First, molecular hydrogen caused growth inhibition of human tongue carcinoma cells HSC-4 and human fibrosarcoma cells HT-1080 but did not compromise growth of normal human tongue epithelial-like cells DOK [14]. Second, hydrogen suppressed the expression of vascular endothelial growth factor (VEGF), a key mediator of tumor angiogenesis, in human lung adenocarcinoma cells A549, which was mediated by downregulation of extracellular signal-regulated kinase (ERK) [15]. Third, hydrogen protected BALB/c mice from developing radiation-induced thymic lymphoma [16]. Elimination of radical oxygen species by hydrogen should reduce a probability of introducing somatic mutations. Unlike other disease models, cancer studies were performed only with cells in two of the three papers. Hydrogen is likely to have a beneficial effect on cancer development by suppressing somatic mutations, but an effect on cancer growth and invasion needs to be analyzed further in detail.

\section{Effects of Molecular Hydrogen on Rodent Models of Neurodegenerative Diseases}

Parkinson's disease is caused by death of dopaminergic neurons at the substantia nigra pars compact of the midbrain and is the second most common neurodegenerative disease after Alzheimer's disease. Parkinson's disease is caused by two mechanisms: excessive oxidative stress and abnormal ubiquitin-proteasome system [17]. The neurotransmitter, dopamine, is a prooxidant by itself and dopaminergic cells are destined to be exposed to high concentrations of radical oxygen species. An abnormal ubiquitin-proteasome system also causes aggregation of insoluble $\alpha$-synuclein in the neuronal cell body that leads to neuronal cell death. We made a rat model of hemi-Parkinson's disease by stereotactically injecting catecholaminergic neurotoxin 6-hydroxydopamine (6-OHDA) in the right striatum [18]. Ad libitum administration of hydrogen-rich water starting one week before surgery completely abolished the development of hemi-Parkinson's symptoms. The number of dopaminergic neurons on the toxin-injected side was reduced to $40.2 \%$ of that on the 
TABLE 1: Sixty-three disease models and human diseases for which beneficial effects of hydrogen have been documented.

\begin{tabular}{|c|c|c|}
\hline Diseases & Species & Administration \\
\hline \multicolumn{3}{|l|}{ Brain } \\
\hline Cerebral infarction $[6,30,55,56]$ & Rodent, human & Gas, saline \\
\hline Cerebral superoxide production [75] & Rodent & Water \\
\hline Restraint-induced dementia [22] & Rodent & Water \\
\hline Alzheimer's disease $[23,24]$ & Rodent & Saline \\
\hline Senile dementia in senescence-accelerated mice [25] & Rodent & Water \\
\hline Parkinson's disease $[18,19]$ & Rodent & Water \\
\hline Hemorrhagic infarction [34] & Rodent & Gas \\
\hline Brain trauma $[76]$ & Rodent & Gas \\
\hline Carbon monoxide intoxication [52] & Rodent & Saline \\
\hline Transient global cerebral ischemia [66] & Rodent & Gas \\
\hline Deep hypothermic circulatory arrest-induced brain damage [57] & Rodent & Saline \\
\hline Surgically induced brain injury [77] & Rodent & Gas \\
\hline \multicolumn{3}{|l|}{ Spinal Cord } \\
\hline Spinal cord injury $[78]$ & Rodent & Saline \\
\hline Spinal cord ischemia/reperfusion [51] & Rabbit & Gas \\
\hline \multicolumn{3}{|l|}{ Eye } \\
\hline Glaucoma $[79]$ & Rodent & Instillation \\
\hline Corneal alkali-burn [61] & Rodent & Instillation \\
\hline \multicolumn{3}{|l|}{ Ear } \\
\hline Hearing loss [80-82] & Tissue, rodent & Medium, water \\
\hline \multicolumn{3}{|l|}{ Lung } \\
\hline Oxygen-induced lung injury $[53,60,83,84]$ & Rodent & Saline \\
\hline Lung transplantation $[85]$ & Rodent & Gas \\
\hline Paraquat-induced lung injury [86] & Rodent & Saline \\
\hline Radiation-induced lung injury [87-89] & Rodent & Water \\
\hline Burn-induced lung injury $[90]$ & Rodent & Saline \\
\hline Intestinal ischemia/reperfusion-induced lung injury [44] & Rodent & Saline \\
\hline \multicolumn{3}{|l|}{ Heart } \\
\hline Acute myocardial infarction $[36,65,91]$ & Rodent & Gas, saline \\
\hline Cardiac transplantation $[46]$ & Rodent & Gas \\
\hline Sleep apnea-induced cardiac hypoxia [48] & Rodent & Gas \\
\hline \multicolumn{3}{|l|}{ Liver } \\
\hline Schistosomiasis-associated chronic liver inflammation [4] & Rodent & Gas \\
\hline Liver ischemia/reperfusion [5] & Rodent & Gas \\
\hline Hepatitis $[43]$ & Rodent & Intestinal gas \\
\hline Obstructive jaundice [47] & Rodent & Saline \\
\hline Carbon tetrachloride-induced hepatopathy [62] & Rodent & Saline \\
\hline Radiation-induced adverse effects for liver tumors [31] & Human & Water \\
\hline \multicolumn{3}{|l|}{ Kidney } \\
\hline Cisplatin-induced nephropathy [92-94] & Rodent & Gas, water \\
\hline Hemodialysis $[20,28]$ & Human & Dialysis solution \\
\hline Kidney transplantation $[95]$ & Rodent & Water \\
\hline Renal ischemia/reperfusion [54] & Rodent & Saline \\
\hline Melamine-induced urinary stone [96] & Rodent & Water \\
\hline Chronic kidney disease [37] & Rodent & Water \\
\hline
\end{tabular}


Table 1: Continued.

\begin{tabular}{|c|c|c|}
\hline Diseases & Species & Administration \\
\hline \multicolumn{3}{|l|}{ Pancreas } \\
\hline Acute pancreatitis [97] & Rodent & Saline \\
\hline \multicolumn{3}{|l|}{ Intestine } \\
\hline Intestinal transplantation $[41,45,59]$ & Rodent & Gas, medium, saline \\
\hline Ulcerative colitis [42] & Rodent & Gas \\
\hline Intestinal ischemia/reperfusion [63] & Rodent & Saline \\
\hline \multicolumn{3}{|l|}{ Blood vessel } \\
\hline Atherosclerosis [98] & Rodent & Water \\
\hline \multicolumn{3}{|l|}{ Muscle } \\
\hline Inflammatory and mitochondrial myopathies [29] & Human & Water \\
\hline \multicolumn{3}{|l|}{ Cartilage } \\
\hline NO-induced cartilage toxicity [38] & Cells & Medium \\
\hline \multicolumn{3}{|l|}{ Metabolism } \\
\hline Diabetes mellitus type I [32] & Rodent & Water \\
\hline Diabetes mellitus type II [26] & Human & Water \\
\hline Metabolic syndrome $[27,99]$ & Human, rodent & Water \\
\hline Diabetes/obesity [33] & Rodent & Water \\
\hline \multicolumn{3}{|l|}{ Perinatal disorders } \\
\hline Neonatal cerebral hypoxia [10-12] & Rodent, pig & Gas, saline \\
\hline Preeclampsia $[58]$ & Rodent & Saline \\
\hline \multicolumn{3}{|l|}{ Inflammation/allergy } \\
\hline Type I allergy [64] & Rodent & Water \\
\hline Sepsis $[100]$ & Rodent & Gas \\
\hline Zymosan-induced inflammation [101] & Rodent & Gas \\
\hline LPS/IFN $\gamma$-induced NO production [67] & Cells & Gas \\
\hline \multicolumn{3}{|l|}{ Cancer } \\
\hline Growth of tongue carcinoma cells [14] & Cells & Medium \\
\hline Lung cancer cells $[15]$ & Cells & Medium \\
\hline Radiation-induced thymic lymphoma [16] & Rodent & Saline \\
\hline \multicolumn{3}{|l|}{ Others } \\
\hline UVB-induced skin injury [49] & Rodent & Bathing \\
\hline Decompression sickness [102] & Rodent & Saline \\
\hline Viability of pluripotent stromal cells [103] & Cells & Gas \\
\hline Radiation-induced cell damage $[104,105]$ & Cells & Medium \\
\hline Oxidized low density lipoprotein-induced cell toxicity [50] & Cells & Medium \\
\hline High glucose-induced oxidative stress [35] & Cells & Medium \\
\hline
\end{tabular}

control side, whereas hydrogen treatment improved the reduction to $83.0 \%$. We also started giving hydrogen-rich water three days after surgery, and hemi-Parkinson's symptoms were again suppressed, but not as much as those observed in pretreated rats. The number of dopaminergic neurons on the toxin-injected side was $76.3 \%$ of that on the control side. Pretreated rats were also sacrificed $48 \mathrm{hrs}$ after toxin injection, and the tyrosine hydroxylase activity at the striatum, where dopaminergic neurons terminate, was decreased in both hydrogen and control groups. This indicated that hydrogen did not directly detoxicate 6-OHDA but exerted a delayed protective effect for dopaminergic cells. Fujita and colleagues also demonstrated a similar prominent effect of hydrogen-rich water on an MPTP-(1-methyl-4phenyl-1,2,3,6-tetrahydropyridine-) induced mouse model of Parkinson's disease [19]. MPTP is a neurotoxin that blocks complex I of the mitochondrial electron transport system and causes Parkinson's disease in mice and humans. 
TABLe 2: Two disease models for which hydrogen has no effect.

\begin{tabular}{lcc}
\hline Diseases & Species & Administration \\
\hline Brain & & \\
\hline $\begin{array}{l}\text { Moderate to severe } \\
\text { neonatal brain hypoxia [9] }\end{array}$ & Rodent & Gas \\
\hline Muscle & & \\
\hline Muscle disuse atrophy [13] & Rodent & Water \\
\hline
\end{tabular}

It is interesting to note that the concentration of hydrogen that they used for the MPTP mice was only $0.08 \mathrm{ppm}(5 \%$ saturation), which is the second lowest among all the trials published to date for rodents and humans. The lowest hydrogen concentration ever tested is $0.048 \mathrm{ppm}$ in the dialysis solution for patients receiving hemodialysis [20].

Alzheimer's disease is the most common neurodegenerative disease and is characterized by abnormal aggregation of $\beta$-amyloid $(A \beta)$ and tau, the large aggregates of which are recognizable as senile plaques and neurofibrillary tangles, respectively [21]. Effects of molecular hydrogen on Alzheimer's disease have been studied in three rodent models. First, Nagata and colleagues made a mouse model of dementia by restricting movement of mice for $10 \mathrm{hrs}$ a day [22]. They analyzed cognitive functions through passive avoidance learning, object recognition tasks, and the Morris water maze and demonstrated that ad libitum administration of hydrogen-rich water efficiently ameliorated cognitive impairment. They also showed that neural proliferation in the dentate gyrus was restored by hydrogen. Second, Li and colleagues made a rat model of Alzheimer's disease by intracerebroventricular injection of $\mathrm{A} \beta 1-42$ [23]. They analyzed cognitive functions by the Morris water maze open field tasks, and electrophysiological measurement of longterm potentiation (LTP) and found that intraperitoneal injection of hydrogen-rich saline for 14 days efficiently ameliorated cognitive decline and preserved LTP. The same team later reported that the protective effects were mediated by suppression of abnormal activation of IL1 $\beta$, JNK, and NF $\kappa \mathrm{B}$ [24]. Third, Gu and colleagues used a senescence-accelerated mouse strain (SAMP8) that exhibits early aging syndromes including impairment in learning ability and memory [25]. Ad libitum administration of hydrogen-rich water for 30 days prevented cognitive decline, which was examined by the Morris water maze. Additionally, ad libitum drinking of hydrogen water for 18 weeks showed efficient amelioration of hippocampal neurodegeneration.

Cerebrovascular diseases are the most frequently reported neurological diseases for which hydrogen has prominent effects. As stated in Section 2, current hydrogen research has broken out after Ohsawa reported a prominent effect of $2-4 \%$ hydrogen for a rat model of left cerebral artery occlusion in 2007 [6].

In addition to neurodegenerative disorders of Parkinson's disease and Alzheimer's disease, effects of molecular hydrogen have been reported in eight other brain diseases listed under the categories of "brain" and "perinatal disorders" in Table 1. The brain consumes a large amount of oxygen and is predisposed to be exposed to a large amount of radical oxygen species especially under pathological conditions. Molecular hydrogen is thus likely to exert a prominent beneficial effect on brain diseases.

\section{Molecular Hydrogen Is Effective for Six Human Diseases}

As in other therapeutic modalities, effects of molecular hydrogen have been tested mostly on rodents but have also been studied in six human diseases. The reported human diseases include diabetes mellitus type II [26], metabolic syndrome [27], hemodialysis [20, 28], inflammatory and mitochondrial myopathies [29], brain stem infarction [30], and radiation-induced adverse effects for liver tumor [31]. These studies are reviewed in detail here. In addition, a therapeutic trial for Parkinson's disease is currently in progress and exhibits favorable responses as far as we know, but the details are not yet disclosed.

First, Kajiyama and colleagues performed a randomized, double-blind, placebo-controlled, crossover study in 30 patients with diabetes mellitus type II and 6 patients with impaired glucose tolerance [26]. The patients consumed either $900 \mathrm{~mL}$ of hydrogen-rich water or placebo water for 8 weeks, with a 12 -week washout period. They measured 13 biomarkers to estimate lipid and glucose metabolisms at baseline and at 8 weeks after hydrogen treatment. All the biomarkers were favorably changed with hydrogen, but statistical significance was observed only in improvement of electronegative charge-modified low-density lipoprotein-(LDL-) cholesterol, small dense LDL, and urinary 8-isoprostanes. In four of six patients with impaired glucose tolerance, hydrogen normalized the oral glucose tolerance test. Lack of statistical significance in their studies was likely due to the small number of patients and the short observation period. Lack of statistical significance, however, may also suggest a less prominent effect in human diabetes mellitus compared to rodent models $[32,33]$.

Second, Nakao and colleagues performed an open-label trial in 20 subjects with potential metabolic syndrome [27]. Hydrogen-rich water was produced by placing a metallic magnesium stick in water, which yielded $0.55-0.65 \mathrm{mM}$ hydrogen water (70-80\% saturation). The participants consumed 1.5-2.0 liters of hydrogen water per day for 8 weeks and showed a $39 \%$ increase in urinary superoxide dismutase (SOD), an enzyme that catalyzes superoxide anion $\left(\mathrm{O}_{2}{ }^{-}\right)$; a $43 \%$ decrease in urinary thiobarbituric acid reactive substances (TBARS), a marker of lipid peroxidation; an 8\% increase in high-density-lipoprotein-(HDL-) cholesterol; a $13 \%$ decrease in total cholesterol/HDL-cholesterol. The aspartate aminotransferase (AST) and alanine transaminase (ALT) levels remained unchanged, whereas the gamma glutamyl transferase (GGT) level was increased by $24 \%$ but was still within a normal range. Although the study was not double blinded and placebo controlled, improvements in biomarkers were much more than those in other hydrogen studies in humans. As this study used a large amount of hydrogen water, the amount of hydrogen might have been a 
critical determinant. Alternatively, excessive hydration might have prevented the participants from excessive food intake.

Third, Nakayama and colleagues performed an openlabel placebo-controlled crossover trial of 12 sessions of hemodialysis in eight patients [28] and an open-label trial of 78 sessions of hemodialysis in 21 patients [20]. In both studies, continuous sessions of hemodialysis with hydrogenrich dialysis solution decreased systolic blood pressure before and after dialysis. In the short-term study, plasma methylguanidine was significantly decreased. In the long-term study, plasma monocyte chemoattractant protein 1 and myeloperoxidase were significantly decreased.

Fourth, we performed an open-label trial of 1.0 liter of hydrogen water per day for 12 weeks in 14 patients with muscular diseases including muscular dystrophies, polymyositis/dermatomyositis, and mitochondrial myopathies, as well as a randomized, double-blind, placebo-controlled, crossover trial of 0.5 liter of hydrogen water or dehydrogenized water per day for 8 weeks in 22 patients with dermatomyositis and mitochondrial myopathies [29]. In the open-label trial, significant improvements were observed in lactateto-pyruvate ratio, fasting blood glucose, serum matrix metalloproteinase-3 (MMP3), and triglycerides. Especially, the lactate-to-pyruvate ratio, which is a sensitive biomarker for the compromised mitochondrial electron transport system, was decreased by $28 \%$ in mitochondrial myopathies. In addition, MMP3, which represents the activity of inflammation, was decreased by $27 \%$ in dermatomyositis. In the double-blind trial, a statistically significant improvement was observed only in serum lactate in mitochondrial myopathies, but lactate-to-pyruvate ratio in mitochondrial myopathies and MMP3 in dermatomyositis were also decreased. Lack of statistical significance with the double-blind study was likely due to the shorter observation period and the lower amount of hydrogen compared to those of the open-label trial.

Fifth, Kang and colleagues performed a randomized placebo-controlled study of 1.5-2.0 liters of $0.55-0.65 \mathrm{mM}$ hydrogen water per day for 6 weeks in 49 patients receiving radiation therapy for malignant liver tumors. Hydrogen suppressed the elevation of total hydroperoxide levels, maintained serum antioxidant capacity, and improved the quality of life (QOL) scores. In particular, hydrogen efficiently prevented loss of appetite. Although the patients were randomly assigned to the hydrogen and placebo groups, the study could not be completely blinded because hydrogen was produced with a metallic magnesium stick, which generated hydrogen bubbles.

Sixth, Ono and colleagues intravenously administered hydrogen along with Edaravone, a clinically approved radical scavenger, in 8 patients with acute brain stem infarction and compared MRI indices of 26 patients who received Edaravone alone [30]. The relative diffusion-weighted images (rDWIs), regional apparent diffusion coefficients (rADCs), and pseudonormalization time of rDWI and $\mathrm{rADC}$ were all improved with the combined infusion of Edaravone and hydrogen.

No adverse effect of hydrogen has been documented in the six human diseases described above. Among the six diseases, the most prominent effect was observed in subjects with metabolic syndrome, who consumed 1.5-2.0 liters of hydrogen water per day [27]. The amount of hydrogen water may be a critical parameter that determines clinical outcome. It is also interesting to note that lipid and glucose metabolisms were analyzed in three studies and all showed favorable responses to hydrogen $[26,27,29]$.

\section{Molecular Bases of Hydrogen Effects}

Effects of hydrogen on various diseases have been attributed to four major molecular mechanisms: a specific scavenging activity of hydroxyl radical, a scavenging activity of peroxynitrite, alterations of gene expressions, and signalmodulating activities. The four mechanisms are not mutually exclusive and some of them may be causally associated with other mechanisms.

The first molecular mechanism identified for hydrogen was its specific scavenging activity of hydroxyl radical [6]. Indeed, oxidative stress markers like 8-OHdG, 4-hydroxyl2-nonenal (4-HNE), malondialdehyde (MDA), and thiobarbituric acid reactive substances (TBARSs) are decreased in all the examined patients and rodents. As hydrogen can easily dissipate in exhalation, hydrogen in drinking water is able to stay in human and rodent bodies in less than 10 min (unpublished data). Hydrogen, however, can bind to glycogen, and the dwell time of hydrogen is prolonged in rat liver after food intake [33]. A question still remains if mice and humans can take a sufficient amount of hydrogen that efficiently scavenges hydroxyl radicals that are continuously generated in normal and disease states.

Another molecular mechanism of hydrogen effect is its peroxynitrite- $\left(\mathrm{ONOO}^{-}-\right)$scavenging activity [6]. Although hydrogen cannot eliminate peroxynitrite as efficiently as hydroxyl radical in vitro [6], hydrogen can efficiently reduce nitric-oxide-(NO-) induced production of nitrotyrosine in rodents [34-38]. NO is a gaseous molecule that also exerts therapeutic effects including relaxation of blood vessels and inhibition of platelet aggregation [39]. NO, however, is also toxic at higher concentrations because $\mathrm{NO}$ leads to $\mathrm{ONOO}^{-}$mediated production of nitrotyrosine, which compromises protein functions. A part of hydrogen effects may thus be attributed to the reduced production of nitrotyrosine.

Expression profiling of rat liver demonstrated that hydrogen has a minimal effect on expression levels of individual genes in normal rats [40]. Gene ontology analysis, however, revealed that oxidoreduction-related genes were upregulated. In disease models of rodents, expression of individual genes and proteins is analyzed. In many disease models, hydrogen downregulated proinflammatory cytokines including tumor necrosis-factor-(TNF-) $\alpha$, interleukin-(IL-) $1 \beta$, IL-6, IL12 , interferon-(IFN-) $\gamma$, and high mobility group box 1 (HMGB1) [4, 23, 24, 36, 41-59]. Hydrogen also downregulated nuclear factors including nuclear factor kappa B $(\mathrm{NF} \kappa \mathrm{B}), \mathrm{JNK}$, and proliferation cell nuclear antigen (PCNA) $[24,44,50,55,60-63]$. Caspases were also downregulated $[10,55-57,62,64,65]$. Other interesting molecules studied to date include vascular endothelial growth factor (VEGF) 
[15]; MMP2 and MMP9 [34]; brain natriuretic peptide [48]; intercellular-adhesion-molecule-1 (ICAM-1) and myeloperoxidase [36]; B-cell lymphoma $2(\mathrm{Bcl} 2)$ and $\mathrm{Bcl} 2$-associated $\mathrm{X}$ protein (Bax) [60]; MMP3 and MMP13 [38]; cyclooxygenase 2 (COX-2), neuronal nitric oxide synthase (nNOS), and connexins 30 and 43 [66]; ionized calcium binding adaptor molecule 1 (Iba1) [52]; fibroblast growth factor 21 (FGF21) [33]. Most molecules, however, are probably passengers that are secondarily changed by hydrogen administration, and some are potentially direct targets of hydrogen effects, which need to be identified in the future.

Using rat RBL-2H3 mast cells, we demonstrated that hydrogen attenuates phosphorylation of FceRI-associated Lyn and its downstream signaling molecules [64]. As phosphorylation of Lyn is again regulated by the downstream signaling molecules and makes a loop of signal transduction pathways, we could not identify the exact target of hydrogen. Our study also demonstrated that hydrogen ameliorates an immediate-type allergic reaction not by radical-scavenging activity but by direct modulation of signaling pathway(s). In addition, using murine RAW264 macrophage cells, we demonstrated that hydrogen reduces LPS/IFN $\gamma$-induced NO production [67]. We found that hydrogen inhibits phosphorylation of ASK1 and its downstream signaling molecules, p38 MAP kinase, JNK, and I $\kappa \mathrm{B} \alpha$ without affecting ROS production by NADPH oxidase. Both studies point to a notion that hydrogen is a gaseous signal modulator. More animal and cells models are expected to be explored to confirm that hydrogen exerts its beneficial effect as a signal modulator.

\section{Enigmas of Hydrogen Effects}

Two enigmas remain to be solved for hydrogen effects. First, no dose-response effect of hydrogen has been observed. Hydrogen has been administered to animals and humans in the forms of hydrogen gas, hydrogen-rich water, hydrogenrich saline, instillation, and dialysis solution (Table 1). Supposing that a $60-\mathrm{kg}$ person drinks $1000 \mathrm{~mL}$ of saturated hydrogen-rich water $(1.6 \mathrm{ppm}$ or $0.8 \mathrm{mM})$ per day, 0.8 mmoles of hydrogen is consumed by the body each day, which is predicted to give rise to a hydrogen concentration of $0.8 \mathrm{mmoles} /(60 \mathrm{~kg} \times 60 \%)=0.022 \mathrm{mM}(2.8 \%$ saturation $=$ $0.022 \mathrm{mM} / 0.8 \mathrm{mM})$. As hydrogen mostly disappears in 10 min by dissipation in exhalation (unpublished data), an individual is exposed to $2.8 \%$ hydrogen only for $10 \mathrm{~min}$. On the other hand, when a person is placed in a $2 \%$ hydrogen environment for $24 \mathrm{hrs}$, body water is predicted to become $2 \%$ saturation $(0.016 \mathrm{mM})$. Even if we suppose that the hydrogen concentration after drinking hydrogen water remains the same for $10 \mathrm{~min}$, areas under the curves of hydrogen water and $2 \%$ hydrogen gas are $0.022 \mathrm{mM} \times 1 / 6 \mathrm{hrs}$ and $0.016 \mathrm{mM} \times 24 \mathrm{hrs}$, respectively. Thus, the amount of hydrogen given by $2 \%$ hydrogen gas should be 104 or more times higher than that given by drinking hydrogen water. In addition, animals and patients are usually not able to drink $100 \%$-saturated hydrogen water. If the hydrogen concentra- tion is $72 \%$ of the saturation level, the peak concentrations achieved by drinking hydrogen water and $2 \%$ hydrogen gas should be identical $(0.022 \mathrm{mM} \times 72 \%=0.016 \mathrm{mM})$. Nevertheless, hydrogen water is as effective as, or sometimes more effective than, hydrogen gas. In addition, orally taken hydrogen can be readily distributed in the stomach, intestine, liver, heart, and lung but is mostly lost in exhalation. Thus, hydrogen concentrations in the arteries are predicted to be very low. Nevertheless, marked hydrogen effects are observed in the brain, spinal cord, kidney, pancreas muscle, and cartilage, where hydrogen is carried via arteries.

The second enigma is intestinal production of hydrogen gas in rodents and humans. Although no mammalian cells can produce hydrogen endogenously, hydrogen is produced by intestinal bacteria carrying hydrogenase in both rodents and humans. We humans are able to make a maximum of 12 liters of hydrogen in our intestines [68, 69]. Specificpathogen-free (SPF) animals are different from aseptic animals and carry intestinal bacteria that produce hydrogen. The amount of hydrogen taken by water or gas is much less than that produced by intestinal bacteria, but the exogenously administered hydrogen demonstrates a prominent effect. In a mouse model of Concanavalin A-induced hepatitis, Kajiya and colleagues killed intestinal bacteria by prescribing a cocktail of antibiotics [43]. Elimination of intestinal hydrogen worsened hepatitis. Restitution of a hydrogenase-negative strain of $E$. coli had no effects, whereas that of a hydrogenase-positive strain of E. coli ameliorated hepatitis. This is the only report that addressed a beneficial effect of intestinal bacteria, and no human study has been reported to date. Kajiya and colleagues also demonstrated that drinking hydrogen-rich water was more effective than the restitution of hydrogenase-positive bacteria. If intestinal hydrogen is as effective as the other hydrogen administration methods, we can easily increase hydrogen concentrations in our bodies by an $\alpha$-glucosidase inhibitor, acarbose [70], an ingredient of curry, turmeric [71], or a nonabsorbable synthetic disaccharide, lactulose $[68,72,73]$. The enigma of intestinal bacteria thus needs to be solved in the future.

\section{Summary and Conclusions}

Effects of hydrogen have been reported in 63 disease models and human diseases (Table 1). Only two diseases of cerebral infarction and metabolic syndrome have been analyzed in both rodents and humans. Lack of any adverse effects of hydrogen enabled clinical studies even in the absence of animal studies. Some other human studies including Parkinson's disease are currently in progress, and promising effects of hydrogen are expected to emerge for many other human diseases. We also have to elucidate molecular bases of hydrogen effects in detail.

\section{Added Note in Proof}

We recently reported a line of evidence that molecular hydrogen has no dose-response effect in a rat model of Parkinson's disease [74]. 


\section{Acknowledgments}

Works performed in the authors' laboratories were supported by Grants-in-Aid from the MEXT and MHLW of Japan and from the Priority Research Project of Aichi.

\section{References}

[1] G. V. Buxton, C. L. Greenstock, W. P. Helman, and A. B. Ross, "Critical view of rate constants for reactions of hydrated electrons, hydrogen atoms and hydroxyl radicals $\left(\bullet \mathrm{OH} / \bullet \mathrm{OH}^{-}\right)$in aqueous solution," Journal of Physical and Chemical Reference Data, vol. 17, pp. 513-886, 1988.

[2] Y. Chuai, F. Gao, B. Li et al., "Hydrogen-rich saline attenuates radiation-induced male germ cell loss in mice through reducing hydroxyl radicals," Biochemical Journal, vol. 442, pp. 49-56, 2012.

[3] V. Lafay, P. Barthelemy, B. Comet, Y. Frances, and Y. Jammes, "ECG changes during the experimental human dive HYDRA 10 (71 atm/7,200 kPa)," Undersea \& Hyperbaric Medicine, vol. 22, no. 1, pp. 51-60, 1995.

[4] B. Gharib, S. Hanna, O. M. S. Abdallahi, H. Lepidi, B. Gardette, and M. De Reggi, "Anti-inflammatory properties of molecular hydrogen: investigation on parasite-induced liver inflammation," Comptes Rendus de l'Academie des SciencesSerie III, vol. 324, no. 8, pp. 719-724, 2001.

[5] K. I. Fukuda, S. Asoh, M. Ishikawa, Y. Yamamoto, I. Ohsawa, and S. Ohta, "Inhalation of hydrogen gas suppresses hepatic injury caused by ischemia/reperfusion through reducing oxidative stress," Biochemical and Biophysical Research Communications, vol. 361, no. 3, pp. 670-674, 2007.

[6] I. Ohsawa, M. Ishikawa, K. Takahashi et al., "Hydrogen acts as a therapeutic antioxidant by selectively reducing cytotoxic oxygen radicals," Nature Medicine, vol. 13, no. 6, pp. 688-694, 2007.

[7] C. S. Huang, T. Kawamura, Y. Toyoda, and A. Nakao, "Recent advances in hydrogen research as a therapeutic medical gas," Free Radical Research, vol. 44, no. 9, pp. 971-982, 2010.

[8] S. Ohta, "Recent progress toward hydrogen medicine: potential of molecular hydrogen for preventive and therapeutic applications," Current Pharmaceutical Design, vol. 17, pp. 2241-2252, 2011.

[9] G. A. Matchett, N. Fathali, Y. Hasegawa et al., "Hydrogen gas is ineffective in moderate and severe neonatal hypoxiaischemia rat models," Brain Research, vol. 1259, pp. 90-97, 2009.

[10] J. Cai, Z. Kang, W. W. Liu et al., "Hydrogen therapy reduces apoptosis in neonatal hypoxia-ischemia rat model," Neuroscience Letters, vol. 441, no. 2, pp. 167-172, 2008.

[11] F. Domoki, O. Oláh, A. Zimmermann et al., "Hydrogen is neuroprotective and preserves cerebrovascular reactivity in asphyxiated newborn pigs," Pediatric Research, vol. 68, no. 5, pp. 387-392, 2010.

[12] J. M. Cai, Z. Kang, K. Liu et al., "Neuroprotective effects of hydrogen saline in neonatal hypoxia-ischemia rat model," Brain Research, vol. 1256, pp. 129-137, 2009.

[13] R. Fujita, Y. Tanaka, Y. Saihara et al., "Effect of molecular hydrogen saturated alkaline electrolyzed water on disuse muscle atrophy in gastrocnemius muscle," Journal of Physiological Anthropology, vol. 30, pp. 195-201, 2011.

[14] Y. Saitoh, H. Okayasu, L. Xiao, Y. Harata, and N. Miwa, "Neutral pH hydrogen-enriched electrolyzed water achieves tumor-preferential clonal growth inhibition over normal cells and tumor invasion inhibition concurrently with intracellular oxidant repression," Oncology Research, vol. 17, no. 6, pp. 247-255, 2008.

[15] J. Ye, Y. Li, T. Hamasaki et al., "Inhibitory effect of electrolyzed reduced water on tumor angiogenesis," Biological and Pharmaceutical Bulletin, vol. 31, no. 1, pp. 19-26, 2008.

[16] L. Zhao, C. Zhou, J. Zhang et al., "Hydrogen protects mice from radiation induced thymic lymphoma in BALB/c mice," International Journal of Biological Sciences, vol. 7, no. 3, pp. 297-300, 2011.

[17] A. H. Schapira, "Mitochondria in the aetiology and pathogenesis of Parkinson's disease," The Lancet Neurology, vol. 7, no. 1, pp. 97-109, 2008.

[18] Y. Fu, M. Ito, Y. Fujita et al., "Molecular hydrogen is protective against 6-hydroxydopamine-induced nigrostriatal degeneration in a rat model of Parkinson's disease," Neuroscience Letters, vol. 453, no. 2, pp. 81-85, 2009.

[19] K. Fujita, T. Seike, N. Yutsudo et al., "Hydrogen in drinking water reduces dopaminergic neuronal loss in the 1-methyl-4phenyl-1,2,3,6-tetrahydropyridine mouse model of Parkinson's disease," PLoS ONE, vol. 4, no. 9, Article ID e7247, 2009.

[20] M. Nakayama, H. Nakano, H. Hamada, N. Itami, R. Nakazawa, and S. Ito, "A novel bioactive haemodialysis system using dissolved dihydrogen $\left(\mathrm{H}_{2}\right)$ produced by water electrolysis: a clinical trial," Nephrology Dialysis Transplantation, vol. 25, no. 9, pp. 3026-3033, 2010.

[21] M. Jucker and L. C. Walker, "Pathogenic protein seeding in Alzheimer disease and other neurodegenerative disorders," Annals of Neurology, vol. 70, pp. 532-540, 2011.

[22] K. Nagata, N. Nakashima-Kamimura, T. Mikami, I. Ohsawa, and S. Ohta, "Consumption of molecular hydrogen prevents the stress-induced impairments in hippocampus-dependent learning tasks during chronic physical restraint in mice," Neuropsychopharmacology, vol. 34, no. 2, pp. 501-508, 2009.

[23] J. Li, C. Wang, J. H. Zhang, J. M. Cai, Y. P. Cao, and X. J. Sun, "Hydrogen-rich saline improves memory function in a rat model of amyloid-beta-induced Alzheimer's disease by reduction of oxidative stress," Brain Research, vol. 1328, pp. 152-161, 2010.

[24] C. Wang, J. Li, Q. Liu et al., "Hydrogen-rich saline reduces oxidative stress and inflammation by inhibit of JNK and NF- $\kappa \mathrm{B}$ activation in a rat model of amyloid-beta-induced Alzheimer's disease," Neuroscience Letters, vol. 491, no. 2, pp. 127-132, 2011.

[25] Y. Gu, C. S. Huang, T. Inoue et al., "Drinking hydrogen water ameliorated cognitive impairment in senescence-accelerated mice," Journal of Clinical Biochemistry and Nutrition, vol. 46, no. 3, pp. 269-276, 2010.

[26] S. Kajiyama, G. Hasegawa, M. Asano et al., "Supplementation of hydrogen-rich water improves lipid and glucose metabolism in patients with type 2 diabetes or impaired glucose tolerance," Nutrition Research, vol. 28, no. 3, pp. 137143, 2008.

[27] A. Nakao, Y. Toyoda, P. Sharma, M. Evans, and N. Guthrie, "Effectiveness of hydrogen rich water on antioxidant status of subjects with potential metabolic syndrome-an open label pilot study," Journal of Clinical Biochemistry and Nutrition, vol. 46, no. 2, pp. 140-149, 2010.

[28] M. Nakayama, S. Kabayama, H. Nakano et al., "Biological effects of electrolyzed water in hemodialysis," Nephron, vol. 112, no. 1, pp. C9-C15, 2009. 
[29] M. Ito, T. Ibi, K. Sahashi, M. Ichihara, and K. Ohno, "Open-label trial and randomized, double-blind, placebocontrolled, crossover trial of hydrogen-enriched water for mitochondrial and inflammatory myopathies," Medical Gas Research, vol. 1, article 24, 2011.

[30] H. Ono, Y. Nishijima, N. Adachi et al., "Improved brain MRI indices in the acute brain stem infarct sites treated with hydroxyl radical scavengers, Edaravone and hydrogen, as compared to Edaravone alone. A non-controlled study," Medical Gas Research, vol. 1, article 12, 2011.

[31] K. M. Kang, Y. N. Kang, I. B. Choi et al., "Effects of drinking hydrogen-rich water on the quality of life of patients treated with radiotherapy for liver tumors," Medical Gas Research, vol. 1, article 11, 2011.

[32] Y. Li, T. Hamasaki, N. Nakamichi et al., "Suppressive effects of electrolyzed reduced water on alloxan-induced apoptosis and type 1 diabetes mellitus," Cytotechnology, vol. 63, no. 2, pp. 119-131, 2011.

[33] N. Kamimura, K. Nishimaki, I. Ohsawa, and S. Ohta, "Molecular hydrogen improves obesity and diabetes by inducing hepatic FGF21 and stimulating energy metabolism in db/db mice," Obesity, vol. 19, no. 7, pp. 1396-1403, 2011.

[34] C. H. Chen, A. Manaenko, Y. Zhan et al., "Hydrogen gas reduced acute hyperglycemia-enhanced hemorrhagic transformation in a focal ischemia rat model," Neuroscience, vol. 169, no. 1, pp. 402-414, 2010.

[35] P. Yu, Z. Wang, X. Sun et al., "Hydrogen-rich medium protects human skin fibroblasts from high glucose or mannitol induced oxidative damage," Biochemical and Biophysical Research Communications, vol. 409, no. 2, pp. 350-355, 2011.

[36] Y. Zhang, Q. Sun, B. He, J. Xiao, Z. Wang, and X. Sun, "Antiinflammatory effect of hydrogen-rich saline in a rat model of regional myocardial ischemia and reperfusion," International Journal of Cardiology, vol. 148, no. 1, pp. 91-95, 2011.

[37] W. J. Zhu, M. Nakayama, T. Mori et al., "Intake of water with high levels of dissolved hydrogen $\left(\mathrm{H}_{2}\right)$ suppresses ischemiainduced cardio-renal injury in Dahl salt-sensitive rats," Nephrology Dialysis Transplantation, vol. 26, no. 7, pp. 2112-2118, 2011.

[38] T. Hanaoka, N. Kamimura, T. Yokota, S. Takai, and S. Ohta, "Molecular hydrogen protects chondrocytes from oxidative stress and indirectly alters gene expressions through reducing peroxynitrite derived from nitric oxide," Medical Gas Research, vol. 1, article 18, 2011.

[39] D. D. Thomas, L. A. Ridnour, J. S. Isenberg et al., "The chemical biology of nitric oxide: implications in cellular signaling," Free Radical Biology and Medicine, vol. 45, no. 1, pp. 18-31, 2008.

[40] Y. Nakai, B. Sato, S. Ushiama, S. Okada, K. Abe, and S. Arai, "Hepatic oxidoreduction-related genes are upregulated by administration of hydrogen-saturated drinking water," Bioscience, Biotechnology and Biochemistry, vol. 75, no. 4, pp. 774-776, 2011.

[41] B. M. Buchholz, D. J. Kaczorowski, R. Sugimoto et al., "Hydrogen inhalation ameliorates oxidative stress in transplantation induced intestinal graft injury," American Journal of Transplantation, vol. 8, no. 10, pp. 2015-2024, 2008.

[42] M. Kajiya, M. J. B. Silva, K. Sato, K. Ouhara, and T. Kawai, "Hydrogen mediates suppression of colon inflammation induced by dextran sodium sulfate," Biochemical and Biophysical Research Communications, vol. 386, no. 1, pp. 11-15, 2009.

[43] M. Kajiya, K. Sato, M. J. B. Silva et al., "Hydrogen from intestinal bacteria is protective for Concanavalin A-induced hepatitis," Biochemical and Biophysical Research Communications, vol. 386, no. 2, pp. 316-321, 2009.

[44] Y. F. Mao, X. F. Zheng, J. M. Cai et al., "Hydrogenrich saline reduces lung injury induced by intestinal ischemia/reperfusion in rats," Biochemical and Biophysical Research Communications, vol. 381, no. 4, pp. 602-605, 2009.

[45] X. Zheng, Y. Mao, J. Cai et al., "Hydrogen-rich saline protects against intestinal ischemia/reperfusion injury in rats," Free Radical Research, vol. 43, no. 5, pp. 478-484, 2009.

[46] A. Nakao, D. J. Kaczorowski, Y. Wang et al., "Amelioration of rat cardiac cold ischemia/reperfusion injury with inhaled hydrogen or carbon monoxide, or both," Journal of Heart and Lung Transplantation, vol. 29, no. 5, pp. 544-553, 2010.

[47] Q. Liu, W. F. Shen, H. Y. Sun et al., "Hydrogen-rich saline protects against liver injury in rats with obstructive jaundice," Liver International, vol. 30, no. 7, pp. 958-968, 2010.

[48] T. Hayashi, T. Yoshioka, K. Hasegawa et al., "Inhalation of hydrogen gas attenuates left ventricular remodeling induced by intermittent hypoxia in mice," American Journal of Physiology, vol. 301, pp. H1062-H1069, 2011.

[49] K. S. Yoon, X. Z. Huang, Y. S. Yoon et al., "Histological study on the effect of electrolyzed reduced water-bathing on UVB radiation-induced skin injury in hairless mice," Biological and Pharmaceutical Bulletin, vol. 34, pp. 1671-1677, 2011.

[50] G. Song, H. Tian, J. Liu, H. Zhang, X. Sun, and S. Qin, " $\mathrm{H}_{2}$ inhibits TNF- $\alpha$-induced lectin-like oxidized LDL receptor1 expression by inhibiting nuclear factor $\kappa \mathrm{B}$ activation in endothelial cells," Biotechnology Letters, vol. 33, no. 9, pp. 1715-1722, 2011.

[51] Y. Huang, K. Xie, J. Li et al., "Beneficial effects of hydrogen gas against spinal cord ischemia-reperfusion injury in rabbits," Brain Research, vol. 1378, pp. 125-136, 2011.

[52] Q. Sun, J. Cai, J. Zhou et al., "Hydrogen-rich saline reduces delayed neurologic sequelae in experimental carbon monoxide toxicity," Critical Care Medicine, vol. 39, no. 4, pp. 765769, 2011.

[53] Q. A. Sun, J. Cai, S. Liu et al., "Hydrogen-rich saline provides protection against hyperoxic lung injury," Journal of Surgical Research, vol. 165, no. 1, pp. e43-e49, 2011.

[54] F. Wang, G. Yu, S. Y. Liu et al., "Hydrogen-rich saline protects against renal ischemia/reperfusion injury in rats," Journal of Surgical Research, vol. 167, no. 2, pp. e339-e344, 2011.

[55] Q. Ji, K. Hui, L. Zhang, X. Sun, W. Li, and M. Duan, "The effect of hydrogen-rich saline on the brain of rats with transient ischemia," Journal of Surgical Research, vol. 168, no. 1, pp. e95-e101, 2011.

[56] Y. Liu, W. Liu, X. Sun et al., "Hydrogen saline offers neuroprotection by reducing oxidative stress in a focal cerebral ischemia-reperfusion rat model," Medical Gas Research, vol. 1, article 15, 2011.

[57] L. Shen, J. Wang, K. Liu et al., "Hydrogen-rich saline is cerebroprotective in a rat model of deep hypothermic circulatory arrest," Neurochemical Research, vol. 36, no. 8, pp. 1501-1511, 2011.

[58] X. Yang, L. Guo, X. Sun, X. Chen, and X. Tong, "Protective effects of hydrogen-rich saline in preeclampsia rat model," Placenta, vol. 32, pp. 681-686, 2011.

[59] B. M. Buchholz, K. Masutani, T. Kawamura et al., "Hydrogen-enriched preservation protects the isogeneic intestinal graft and amends recipient gastric function during transplantation," Transplantation, vol. 92, pp. 985-992, 2011.

[60] C. S. Huang, T. Kawamura, X. Peng et al., "Hydrogen inhalation reduced epithelial apoptosis in ventilator-induced lung injury via a mechanism involving nuclear factor-kappa B 
activation," Biochemical and Biophysical Research Communications, vol. 408, no. 2, pp. 253-258, 2011.

[61] M. Kubota, S. Shimmura, S. Kubota et al., "Hydrogen and $\mathrm{N}$-acetyl-L-cysteine rescue oxidative stress-induced angiogenesis in a mouse corneal alkali-burn model," Investigative Ophthalmology and Visual Science, vol. 52, no. 1, pp. 427-433, 2011.

[62] H. Sun, L. Chen, W. Zhou et al., "The protective role of hydrogen-rich saline in experimental liver injury in mice," Journal of Hepatology, vol. 54, no. 3, pp. 471-480, 2011.

[63] H. Chen, Y. P. Sun, P. F. Hu et al., "The effects of hydrogenrich saline on the contractile and structural changes of intestine induced by ischemia-reperfusion in rats," Journal of Surgical Research, vol. 167, no. 2, pp. 316-322, 2011.

[64] T. Itoh, Y. Fujita, M. Ito et al., "Molecular hydrogen suppresses FceRI-mediated signal transduction and prevents degranulation of mast cells," Biochemical and Biophysical Research Communications, vol. 389, no. 4, pp. 651-656, 2009.

[65] Q. Sun, Z. Kang, J. Cai et al., "Hydrogen-rich saline protects myocardium against ischemia/reperfusion injury in rats," Experimental Biology and Medicine, vol. 234, no. 10, pp. 1212-1219, 2009.

[66] M. Hugyecz, É. Mracskó, P. Hertelendy, E. Farkas, F. Domoki, and F. Bari, "Hydrogen supplemented air inhalation reduces changes of prooxidant enzyme and gap junction protein levels after transient global cerebral ischemia in the rat hippocampus," Brain Research, vol. 1404, pp. 31-38, 2011.

[67] T. Itoh, N. Hamada, R. Terazawa et al., "Molecular hydrogen inhibits lipopolysaccharide/interferon $\gamma$-induced nitric oxide production through modulation of signal transduction in macrophages," Biochemical and Biophysical Research Communications, vol. 411, no. 1, pp. 143-149, 2011.

[68] S. U. Christl, P. R. Murgatroyd, G. R. Gibson, and J. H. Cummings, "Production, metabolism, and excretion of hydro-gen in the large intestine," Gastroenterology, vol. 102, no. 4, pp. 1269-1277, 1992.

[69] A. Strocchi and M. D. Levitt, "Maintaining intestinal $\mathrm{H}_{2}$ balance: credit the colonic bacteria," Gastroenterology, vol. 102, no. 4, pp. 1424-1426, 1992.

[70] Y. Suzuki, M. Sano, K. Hayashida, I. Ohsawa, S. Ohta, and K. Fukuda, "Are the effects of $\alpha$-glucosidase inhibitors on cardiovascular events related to elevated levels of hydrogen gas in the gastrointestinal tract?" FEBS Letters, vol. 583, no. 13, pp. 2157-2159, 2009.

[71] A. Shimouchi, K. Nose, M. Takaoka, H. Hayashi, and T. Kondo, "Effect of dietary turmeric on breath hydrogen," Digestive Diseases and Sciences, vol. 54, no. 8, pp. 1725-1729, 2009.

[72] G. R. Corazza, M. Sorge, A. Strocchi et al., "Non-absorbable antibiotics and small bowel bacterial overgrowth," Italian Journal of Gastroenterology, vol. 24, no. 9, pp. 4-9, 1992.

[73] X. Chen, Q. Zuo, Y. Hai, and X. J. Sun, "Lactulose: an indirect antioxidant ameliorating inflammatory bowel disease by increasing hydrogen production," Medical Hypotheses, vol. 76, no. 3, pp. 325-327, 2011.

[74] M. Ito, M. Hirayama, K. Yamai et al., "Drinking hydrogen water and intermittent hydrogen gas exposure, but not lactulose or continuous hydrogen gas exposure, prevent 6hydorxydopamine-induced Parkinson's disease in rats," Medical Gas Research, vol. 2, article 15, 2012.

[75] Y. Sato, S. Kajiyama, A. Amano et al., "Hydrogen-rich pure water prevents superoxide formation in brain slices of vitamin C-depleted SMP30/GNL knockout mice," Biochemical and Biophysical Research Communications, vol. 375, no. 3, pp. 346-350, 2008.

[76] X. Ji, W. Liu, K. Xie et al., "Beneficial effects of hydrogen gas in a rat model of traumatic brain injury via reducing oxidative stress," Brain Research, vol. 1354, pp. 196-205, 2010.

[77] J. M. Eckermann, W. Chen, V. Jadhav et al., "Hydrogen is neuroprotective against surgically induced brain injury," Medical Gas Research, vol. 1, article 7, 2011.

[78] C. Chen, Q. Chen, Y. Mao et al., "Hydrogen-rich saline protects against spinal cord injury in rats," Neurochemical Research, vol. 35, no. 7, pp. 1111-1118, 2010.

[79] H. Oharazawa, T. Igarashi, T. Yokota et al., "Protection of the retina by rapid diffusion of hydrogen: administration of hydrogen-loaded eye drops in retinal ischemia-reperfusion injury," Investigative Ophthalmology and Visual Science, vol. 51, no. 1, pp. 487-492, 2010.

[80] Y. S. Kikkawa, T. Nakagawa, R. T. Horie, and J. Ito, "Hydrogen protects auditory hair cells from free radicals," NeuroReport, vol. 20, no. 7, pp. 689-694, 2009.

[81] A. Taura, Y. S. Kikkawa, T. Nakagawa, and J. Ito, "Hydrogen protects vestibular hair cells from free radicals," Acta OtoLaryngologica, vol. 130, no. 563, pp. 95-100, 2010.

[82] Y. Lin, A. Kashio, T. Sakamoto, K. Suzukawa, A. Kakigi, and T. Yamasoba, "Hydrogen in drinking water attenuates noiseinduced hearing loss in guinea pigs," Neuroscience Letters, vol. 487, no. 1, pp. 12-16, 2011.

[83] J. Zheng, K. Liu, Z. Kang et al., "Saturated hydrogen saline protects the lung against oxygen toxicity," Undersea and Hyperbaric Medicine, vol. 37, no. 3, pp. 185-192, 2010.

[84] C. S. Huang, T. Kawamura, S. Lee et al., "Hydrogen inhalation ameliorates ventilator-induced lung injury," Critical Care, vol. 14, no. 6, article R234, 2010.

[85] T. Kawamura, C. S. Huang, N. Tochigi et al., "Inhaled hydrogen gas therapy for prevention of lung transplant-induced ischemia/reperfusion injury in rats," Transplantation, vol. 90, no. 12, pp. 1344-1351, 2010.

[86] S. Liu, K. Liu, Q. Sun et al., "Consumption of hydrogen water reduces paraquat-induced acute lung injury in rats," Journal of Biomedicine and Biotechnology, vol. 2011, Article ID 305086, 7 pages, 2011.

[87] L. Qian, F. Cao, J. Cui et al., "The potential cardioprotective effects of hydrogenin irradiated mice," Journal of Radiation Research, vol. 51, no. 6, pp. 741-747, 2010.

[88] Y. Terasaki, I. Ohsawa, M. Terasaki et al., "Hydrogen therapy attenuates irradiation-induced lung damage by reducing oxidative stress," American Journal of Physiology, vol. 301, pp. L415-L426, 2011.

[89] Y. Chuai, L. Zhao, J. Ni et al., "A possible prevention strategy of radiation pneumonitis: combine radiotherapy with aerosol inhalation of hydrogen-rich solution," Medical Science Monitor, vol. 17, no. 4, pp. 1-4, 2011.

[90] Y. Fang, X. J. Fu, C. Gu et al., "Hydrogen-rich saline protects against acute lung injury induced by extensive burn in rat model," Journal of Burn Care and Research, vol. 32, no. 3, pp. e82-e91, 2011.

[91] K. Hayashida, M. Sano, I. Ohsawa et al., "Inhalation of hydrogen gas reduces infarct size in the rat model of myocardial ischemia-reperfusion injury," Biochemical and Biophysical Research Communications, vol. 373, no. 1, pp. 3035, 2008.

[92] N. Nakashima-Kamimura, T. Mori, I. Ohsawa, S. Asoh, and S. Ohta, "Molecular hydrogen alleviates nephrotoxicity 
induced by an anti-cancer drug cisplatin without compromising anti-tumor activity in mice," Cancer Chemotherapy and Pharmacology, vol. 64, no. 4, pp. 753-761, 2009.

[93] A. Kitamura, S. Kobayashi, T. Matsushita, H. Fujinawa, and K. Murase, "Experimental verification of protective effect of hydrogen-rich water against cisplatin-induced nephrotoxicity in rats using dynamic contrast-enhanced CT, British Journal of Radiology, vol. 83, no. 990, pp. 509-514, 2010.

[94] T. Matsushita, Y. Kusakabe, A. Kitamura, S. Okada, and K. Murase, "Investigation of protective effect of hydrogenrich water against cisplatin-induced nephrotoxicity in rats using blood oxygenation level-dependent magnetic resonance imaging," Japanese Journal of Radiology, vol. 29, pp. 503-512, 2011.

[95] J. S. Cardinal, J. Zhan, Y. Wang et al., "Oral hydrogen water prevents chronic allograft nephropathy in rats," Kidney International, vol. 77, no. 2, pp. 101-109, 2010.

[96] Y. S. Yoon, D. H. Kim, S. K. Kim et al., "The melamine excretion effect of the electrolyzed reduced water in melamine-fed mice," Food and Chemical Toxicology, vol. 49, no. 8, pp. 18141819, 2011.

[97] H. Chen, Y. P. Sun, Y. Li et al., "Hydrogen-rich saline ameliorates the severity of l-arginine-induced acute pancreatitis in rats," Biochemical and Biophysical Research Communications, vol. 393, no. 2, pp. 308-313, 2010.

[98] I. Ohsawa, K. Nishimaki, K. Yamagata, M. Ishikawa, and S. Ohta, "Consumption of hydrogen water prevents atherosclerosis in apolipoprotein E knockout mice," Biochemical and Biophysical Research Communications, vol. 377, no. 4, pp. 1195-1198, 2008.

[99] M. Hashimoto and M. Katakura, "Effects of hydrogen-rich water on abnormalities in a SHR.Cg-Leprcp/NDmcr rat-a metabolic syndrome rat model," Medical Gas Research, vol. 1, article 26, 2011.

[100] K. Xie, Y. Yu, Y. Pei et al., "Protective effects of hydrogen gas on murine polymicrobial sepsis via reducing oxidative stress and HMGB1 release," Shock, vol. 34, no. 1, pp. 90-97, 2010.

[101] K. L. Xie, Y. H. Yu, Z. S. Zhang et al., "Hydrogen gas improves survival rate and organ damage in zymosan-induced generalized inflammation model," Shock, vol. 34, no. 5, pp. 495-501, 2010.

[102] X. X. Ni, Z. Y. Cai, D. F. Fan et al., "Protective effect of hydrogen-rich saline on decompression sickness in rats," Aviation Space and Environmental Medicine, vol. 82, no. 6, pp. 604-609, 2011.

[103] H. Kawasaki, J. Guan, and K. Tamama, "Hydrogen gas treatment prolongs replicative lifespan of bone marrow multipotential stromal cells in vitro while preserving differentiation and paracrine potentials," Biochemical and Biophysical Research Communications, vol. 397, no. 3, pp. 608-613, 2010.

[104] L. R. Qian, F. Cao, J. Cui et al., "Radioprotective effect of hydrogen in cultured cells and mice," Free Radical Research, vol. 44, no. 3, pp. 275-282, 2010.

[105] L. R. Qian, B. L. Li, F. Cao et al., "Hydrogen-rich PBS protects cultured human cells from ionizing radiationinduced cellular damage," Nuclear Technology and Radiation Protection, vol. 25, no. 1, pp. 23-29, 2010. 


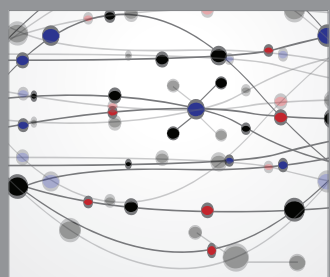

The Scientific World Journal
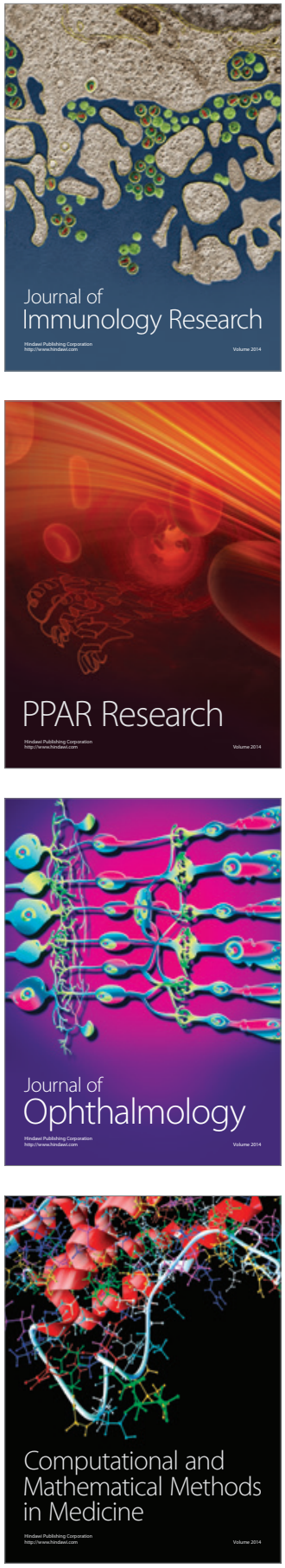

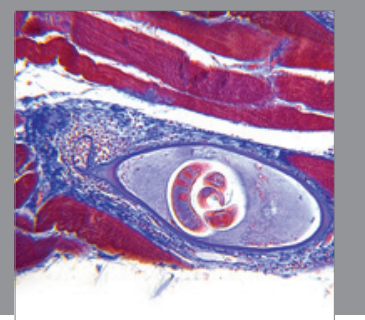

Gastroenterology

Research and Practice
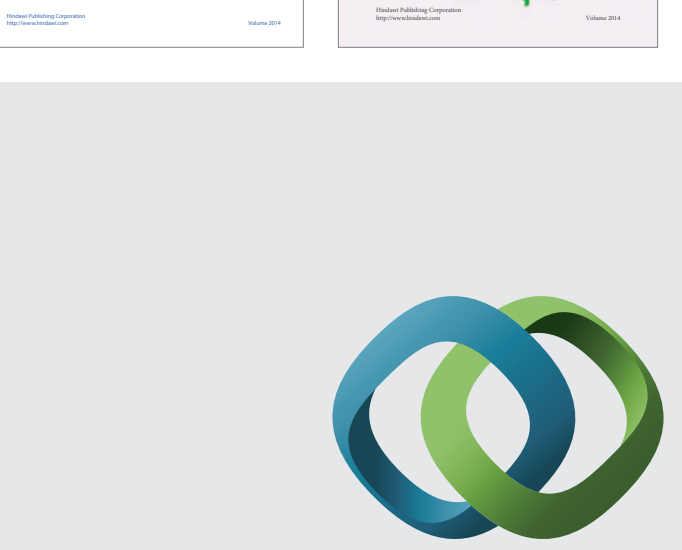

\section{Hindawi}

Submit your manuscripts at

http://www.hindawi.com
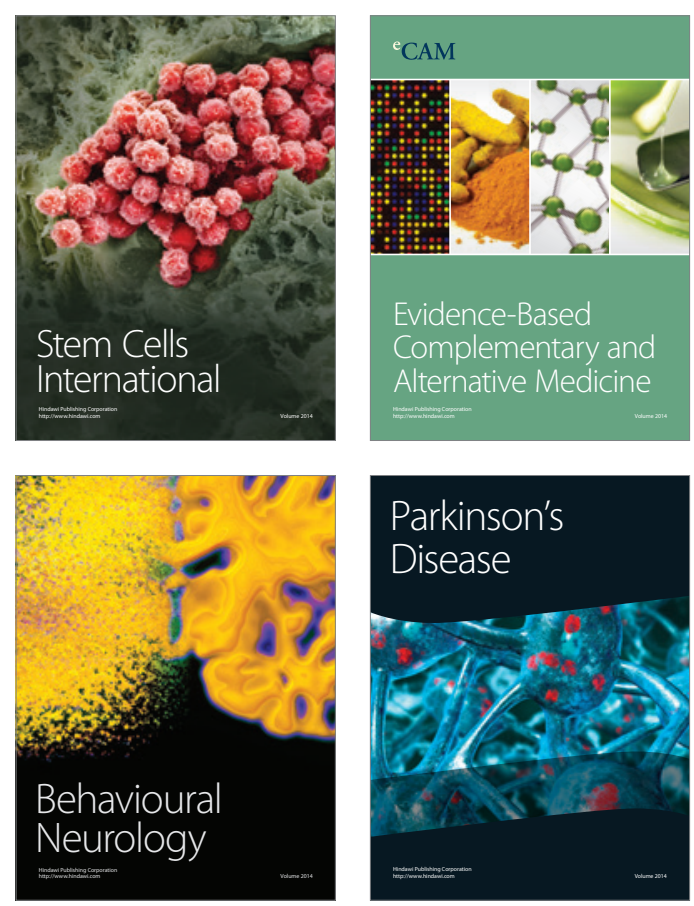

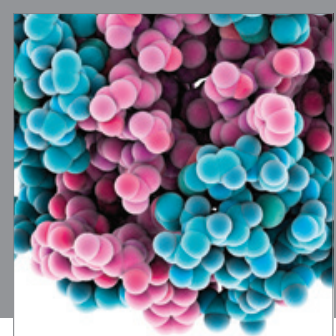

Journal of
Diabetes Research

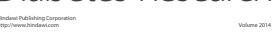

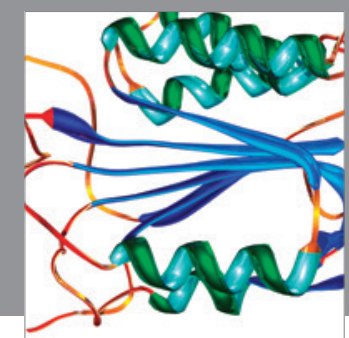

Disease Markers
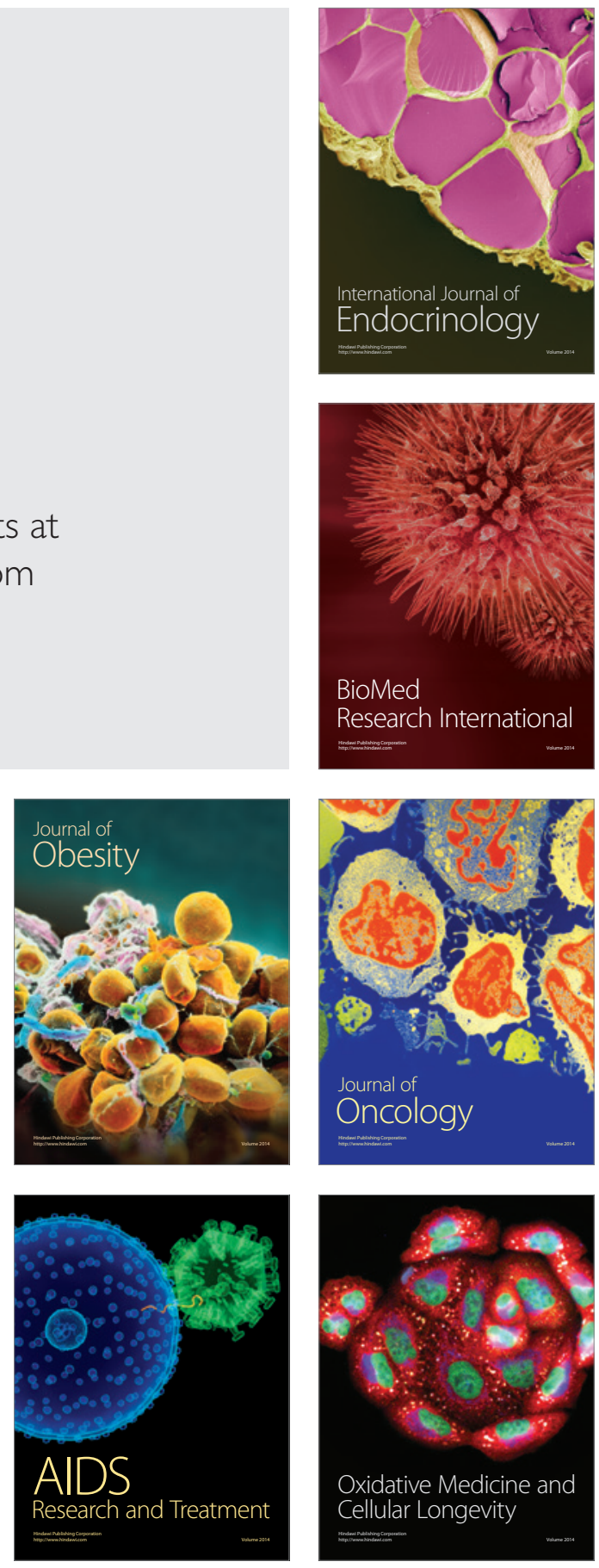